\title{
Amaurosis bilateral como secuela de la intoxicación aguda por metanol: a propósito de un caso.
}

\author{
Bilateral blindness as a consequence of acute methanol \\ intoxication: a case report.
}
N. Olivier Pascual ${ }^{1}$, J. Viéitez Vázquez ${ }^{1}$, A. Arbizu Duralde¹, M. Asencio Durán ${ }^{1}$ y N. Ruiz del Río'

\section{RESUMEN}

La intoxicación por metanol supone una preocupación para la salud pública y ambiental por las acciones selectivas de su metabolito neurotóxico, formaldehído en la retina, nervio óptico y sistema nervioso central. Se presenta un caso de ceguera por metanol y se discute su fisiopatología, diagnóstico y tratamiento.

Pese a que esta neuropatía es rara actualmente, el diagnóstico precoz y el tratamiento adecuado son cruciales para mejorar el pronóstico.

Palabras clave: intoxicación, metílico, neuritis óptica.

\section{ABSTRACT}

Methanol intoxication is an important public health and environmental concern because of the specific actions of its neurotoxic metabolite, formaldehyde, on the retina, optic nerve and central nervous system. We report a case of methanol blindness and discuss its physiopathology, diagnosis and treatment. Although this optic neuropathy is now rare, prompt diagnosis and proper treatment in the acute phase can dramatically improve the prognosis..

Key words: intoxication, methanol, optical neuropathy.

1 Facultativo del Servicio de Oftalmología del Hospital Universitario La Paz (Madrid). 


\section{INTRODUCCIÓN:}

La intoxicación por metanol es una entidad de pronóstico tanto vital como visual pobre. Aunque se producen cada vez menos casos han sido frecuentes a lo largo de la historia episodios de intoxicaciones masivas. En la actualidad obedecen sobre todo a intentos autolíticos o intoxicaciones accidentales. La rápida instauración del tratamiento adecuado es crucial para evitar las secuelas neurológicas y visuales permanentes. Presentamos el caso de una intoxicación aguda por metanol que acabó en ceguera.

\section{CASO CLÍNICO:}

C.M.B., varón de 44 años de edad, es encontrado en su domicilio por sus familiares con un severo cuadro de desorientación y visión borrosa tras consumo abusivo de alcohol los días previos, al parecer en relación con un problema familiar. Se traslada a nuestro centro dónde ingresa en la UVI.

Como antecedentes personales destacan enolismo severo de más de 20 años de evolución, en tratamiento de deshabituación seguido muy irregularmente y cuadro depresivo. Ha sufrido diversas complicaciones médicas de su habito alcohólico (crisis comiciales, hematoma subdural, hemorragia subaracnoidea, gastritis...).

A su ingreso en urgencias el paciente está consciente y orientado y refiere visión borrosa. Los familiares reconocen consumo habitual de alcohol en tratamiento de desintoxicación con pobre cumplimiento e ingesta de benzodiacepinas, con incremento del consumo en los días previos y no descartan la posibilidad de ingestión de otros tóxicos (colonia) ya que el paciente tiene habitualmente restringido el acceso a bebidas alcohólicas.

En la exploración: Tendencia pícnica. Normoconstituido con regular estado de hidratación, no rigidez de nuca, destaca como hallazgo patológico midriasis bilateral arreactiva y disnea II/III, pulso venoso normal, auscultación cardiopulmonar normal. Abdomen blando, depresible, se palpa hígado en límite no doloroso a la palpación, ruidos abdominales normales, reflejos osteotendinosos normales.

En las exploraciones complementarias al ingreso se observa acidosis metabólica severa: GAB: pH 7.। I, pO2 130, pCO2 13, Bicarbonato estándar 7, EB -23. Analítica: Glucosa I 44; Urea 23; creatinina I.20; $\mathrm{Na}^{+}$140; $\mathrm{K}^{+}$4.42. TAC craneal normal. ECG: ritmo sinusal eje horizontalizado sin otras alteraciones.

Sospechando inicialmente una intoxicación benzodiacepínica se traslada a la $\mathrm{UCl}$ y se instaura tratamiento con antagonistas (flumacenilo) y restauración del equilibrio ácido-base.

En posteriores entrevistas con la familia esta reconoce que el paciente tiene restringido el acceso a bebidas alcohólicas y que no descartan consumos do otros tóxicos, en particular colonia, dato que añadido a la dificultad de revertir su acidosis y la nula actividad del flumacenilo sobre el paciente reorientan la sospecha clínica inicial hacia la intoxicación por metanol, instaurándose tratamiento con un competidor metabólico (etanol) y hemodiálisis para intentar la eliminación de metabolitos tóxicos.

Una vez estabilizado se traslada a planta de medicina y se efectúa valoración oftalmológica y psiquiátrica: el paciente no percibe luz y tiene pupilas midriáticas débilmente reactivas. No colabora en la evaluación de la motilidad ocular extrínseca, presenta en el fondo de ojo papilas levemente pálidas sin hemorragia ni edema. Se solicita estudio de PEV. Se descarta intencionalidad autolítica y el paciente reconoce consumo de otras formas de alcohol más económicas. Se le pauta continuar con su tratamiento de deshabituación y seguimiento periódico por su centro de salud mental. 
El paciente es dado de alta 8 días después del ingreso en situación de ceguera legal bilateral con el consiguiente deterioro añadido a su precaria situación socio-familiar.

\section{DISCUSIÓN:}

El alcohol metílico, metanol o alcohol de madera es un líquido claro, incoloro, inflamable con ligero olor a alcohol. Se usa frecuentemente en líquidos de limpiaparabrisas, anticongelantes, como combustible de bombonas de camping y antorchas de soldadura, otros usos incluyen su empleo como disolvente industrial, sustituto de combustibles derivados de petróleo y con frecuencia como desnaturalizante del alcohol etílico.

Los primeros casos descritos de intoxicación por metanol se remontan a 1870 y durante la primera mitad del siglo $X X$ se describieron grandes series de epidemias debidas fundamentalmente al consumo de alcohol adulterado $[1,2]$.

La forma de intoxicación más frecuente hoy en día es bien por ingesta accidental (ya que el metílico es muy parecido al alcohol etílico tanto en su sabor como en olor) o con intencionalidad suicida. Se han descrito de forma aislada otras formas de intoxicación tales como la inhalatoria a partir de disolventes de pinturas, percutánea e incluso la inyección intravenosa [3].

El metanol se absorbe rápidamente y se distribuye en el agua corporal. El máximo nivel en sangre se produce a los 30-90 minutos después de la ingesta. En ausencia de etanol la eliminación renal de metanol sin metabolizarse supone de un 2-5\%, una pequeña cantidad se elimina por vía respiratoria. El aclaramiento depende de la concentración. Con bajos niveles en sangre la vida media de eliminación es de 2 o 3 horas. Cuando los niveles sanguíneos sobrepasan los 300 mg/dl se saturan las enzimas y la vida media de eliminación llega a las 27 horas y la eliminación renal y respiratoria asume una mayor importancia. Durante la terapia con etanol la vida media alcanza unas 30-52 horas. La susceptibilidad es variable según los sujetos pero de 15 a $30 \mathrm{ml}$ pueden bastar para producir una intoxicación severa.

La principal toxicidad del metanol deriva de su conversión hepática en sus metabolitos tóxicos: formaldehído y ácido fórmico. El ácido fórmico es el responsable de la acidosis metabólica profunda que se produce en estas intoxicaciones [4]. Puede aparecer lactato en las fases tardías del metabolismo del metanol y produce hipotensión e hipoxia tisular conduciendo al metabolismo anaeróbico de la glucosa. El folato es un cofactor necesario para la conversión de los formatos a dióxido de carbono. Por este motivo los déficits de folatos (que aparecen con frecuencia en pacientes debilitados o en alcohólicos crónicos) predisponen a un envenenamiento más severo con la ingesta de metanol. Se pensó clásicamente que la producción de formaldehído era la responsable de la toxicidad ocular, y pese a que los niveles de metanol que se encuentran en humor vítreo son similares a los sanguíneos en la actualidad parece claro que la acidosis metabólica y los efectos oculares se deben al acúmulo de ácido fórmico que es capaz de inhibir el complejo citocromo oxidasa de la cadena respiratoria mitocondrial e interferir con la ATPasa sodio-potasio en el nervio óptico lo que conduce a la muerte celular [5]. A nivel del nervio óptico los hallazgos histopatológicos consecuentes son de desmielinización circunscrita con axones preservados $[6,7]$.

La dosis letal de metanol sin tratamiento se estima en unos $80 \mathrm{mg} / \mathrm{dl}$. Las dosis letales publicadas varían considerablemente, así $40 \mathrm{ml}$ de metanol al I5\% ha resultado en muerte mientras que ingestas de 500-600 ml han sobrevivido. Cuando el metanol no es co-ingerido la dosis fatal de metanol se sitúa entre 30-240 ml o aproximadamente l g/kg. La dosis mínima capaz de causar defectos visuales permanentes no ha sido establecida. 
Frecuentemente los primeros síntomas de la intoxicación consisten en embriaguez, somnolencia y vértigo, que aparecen aproximadamente una hora tras la ingesta del metanol y con frecuencia estos síntomas son menores a los que se producen ante dosis similares de alcohol etílico. En la mayoría de los casos estos síntomas iniciales se siguen de un periodo asintomático que puede durar de 6 a 30 horas, especialmente si el metanol se ingiere mezclado con etanol. Así concentraciones de etanol entre 100 y $150 \mathrm{mg} / \mathrm{ml}$ puede retrasar la instauración de los síntomas hasta que se haya metabolizado una cantidad suficiente de etanol como para que el metanol empiece a transformarse en sus metabolitos tóxicos. Sin embargo incluso si el metanol se consume aisladamente pueden transcurrir de 12 a 24 horas hasta que se produzcan concentraciones de metabolitos tóxicos en cantidad suficiente como para producir síntomas. Por ello clásicamente se ha descrito un periodo asintomático que oscila entre 18 y 48 horas. Sin embargo es importante resaltar que esta ausencia de clínica inicial no excluye el posterior desarrollo de toxicidad importante.

Tras el periodo asintomático, los primeros síntomas suelen deberse a la irritación del tracto gastrointestinal: náuseas, vómitos y dolor abdominal que pueden llegar a gastritis (incluso hemorrágica) y pancreatitis. Aparecen mareo y cefalea con frecuencia y característicamente preceden al desarrollo de acidosis metabólica y compromiso visual.

Los síntomas oftalmológicos son variables y van desde visión borrosa o percepción de miodesopsias a la ceguera con midriasis bilateral arreactiva. Cuando la agudeza visual está parcialmente conservada pueden aparecer anomalías en el campo visual tales como escotomas centrales o cecocentrales y en ocasiones restricción concéntrica del campo visual. En el fondo de ojo puede aparecer hiperemia en el nervio óptico o edema de los bordes papilares que puede extenderse a las fibras nerviosas temporales superiores e inferiores. La vascularización retiniana y coroidea así como el resto de la retina son normales.

Otras complicaciones de la intoxicación aguda incluyen convulsiones, coma, ceguera, fallo renal oligúrico, hiper o hipotermia, fallo cardiaco, edema pulmonar, cambios del ECG (fibrilación auricular y cambios en las ondas ST-T), hipotensión, respiración de Kussmaul secundaria a la acidosis y parada respiratoria.

El diagnóstico se basa en la existencia de acidosis metabólica con gap osmolar o aniónico severo y rebelde al tratamiento convencional, que determinará la búsqueda de tóxicos en sangre [8]. Cuando se sospecha una intoxicación por metanol serán del interés los siguientes estudios: agudeza visual basal, electrolitos y osmolaridad sérica, niveles de etanol y metanol, amilasa, función hepática y renal y análisis de orina. Se puede sugerir el diagnóstico mediante técnicas de neuroimagen (fundamentalmente palidez de los ganglios basales) [9], se han descrito en intoxicaciones agudas la necrosis bilateral y simétrica en putamen y hemorragias en sustancia blanca subcortical [10]. A lo largo de la evolución y durante el tratamiento habrá que monitorizar los niveles de metanol, etanol y glucemia.

La evolución dependerá de la severidad de la intoxicación y del retraso en la instauración de medidas terapéuticas. En los casos de intoxicación severa en paciente morirá por insuficiencia respiratoria.

Se han descrito casos de recuperación visual completa a la semana [ I I ] e incluso a las dos semanas [12] de la intoxicación con desaparición del edema papilar. Con mayor frecuencia el pronóstico visual es sombrío con disminución severa y definitiva de agudeza visual y atrofia del nervio óptico en I-2 meses, que se manifiesta como blanqueamiento del nervio óptico de bordes netos sin otra anomalía. En raros casos se ha descrito excavación papilar con desaparición completa del anillo neurorretiniano sin hipertensión intraocular. Este hallazgo sugiere pérdida extensa de células ganglionares. La ausencia inicial de reflejo fotomotor sería signo de mal pronóstico. 
El estudio electrofisiológico de la retina muestra discretas anomalías del tipo de atenuación de la onda b del electrorretinograma por lesión de las células de Muller. Los potenciales evocados visuales están muy alterados y a veces, desaparecen.

El tratamiento debe instaurarse a ser posible en las primeras horas tras la ingestión. Se basa en controlar el equilibrio ácido-base mediante inyección de bicarbonato intravenoso, favorecer la eliminación del agente tóxico por hemodiálisis $[13,14]$, e inhibir la degradación hepática del metanol mediante un neutralizador bien etanol o más recientemente 4 metil pirazona. El tratamiento debe iniciarse con la estabilización de la vía aérea y soporte circulatorio. Si la ingesta tuvo lugar en un tiempo inferior a 6 horas está indicado el lavado gástrico con solución bicarbonatada al 2-10\%. El carbón activo y el purgante salino resultan ineficaces, así como las medidas para forzar la diuresis [15].

En el plano oftalmológico ningún tratamiento ha demostrado fehacientemente su eficacia sin embargo algunos autores proponen el uso de corticoterapia en bolo seguida de dosis orales de I mg/kg día con intención de disminuir el edema papilar, acompañado o no de vitaminoterapia (vitamina BI 100 mg/dia IM) debido a su eficacia en el tratamiento de neuropatías periféricas y centrales por agentes exógenos crónicos [12].

\section{CONCLUSIONES:}

Pese a la reducida incidencia de esta neuropatía óptica en la actualidad su severo pronóstico y la necesidad de un rápido tratamiento hacen que el diagnóstico deba ser lo más precoz posible. Por tanto es necesario plantearse esta posibilidad diagnóstica ante la asociación de signos oftalmológicos bilaterales del tipo de disminución de la agudeza visual severa, midriasis arreactiva, edema papilar junto a clínica de acidosis severa en al contexto de un paciente en estado de embriaguez o con antecedentes de alcoholismo.

\section{BIBLIOGRAFÍA:}

I.- Benton CD, Calhoun FP. The ocular effects of methyl alcohol poisoning: report of a catastrophe involving three hundred and twenty persons. Trans Am Acad Ophtalmol, 1952;56:875-83.

2.- Krivosic V, Vignal-Clermont C, Blain P, Gaudric A. Neuropathie optique bilatérale par intoxication aiguë au methanol. A propos d'un cas. J Fr. Ophtalmol, 2001;24;5:522-6.

3.- Wang Jenn Yeu. Methanol intoxication with retinal injury by intravenous injection. Ann Emerg Med.1999;34:297

4.- Ferrari LA, Arado MG, Nardo CA, Giannuzzi. Post-mortem analysis of formic acid disposition in acute methanol intoxication. Forensic Sci Int. 2003 Apr 23;133(I-2):152-8.

5.- Gonzalez-Quevedo A, Obregón F, Urbina M, Rousso T, Lima L. Effect of chronic metanol administration on amino acids and monoamines in retina, optic nerve, and brain of the rat. Toxicol Appll Pharmacol. 2002 Dec I;:185(2):77-84.

6.- Sharpe JA, Hostovsky M, Rewcastle NB. Methanol ophtic neuropathy: a histopatological study. Neurology. 1982. 32(10):1093-100. 7.- Seme M.T., Summerfelt P., Henry M.M., et al. Formate-induced inhibition of photoreceptor function in methanol intoxication. JPET. 1999. 298(I):361-70.

8.- Weinberg L, Stewart J, Wyatt JP Mathew J. Unexplained drowsiness and progressive visual loss: Methanol poisoning diagnosed at autopsy. Emerg Med. 2003 Feb;15(I):97-9.

9.- Halavaara J, Valane L, Setala K. Neuroimaging supports clinical findings of metanol poisoning. Neuroradiology. 2002 Nov;44(II):924-8.

10.- Cavit BOZ, Sibel $\mathrm{K}$ et al. Bilateral putaminal hemorrhagic infarction and optic atrophy caused by methanol intoxication. Norol Bil. 2000 17:4.

II.- Rossazza C Delplace MP, Boulanger JF, Leboucq F. Significant but transient visual recovery after methanol poisoning. Bull Soc Ophthalmol Fr, 1983;83:545-8.

12.- Rotenstreich $Y$ et al. Late treatment of methanol blindness. $\mathrm{Br}$ J Ophthalmol, 1997;81:416-7.

13.- Osterloh JD, Pond SM, Grady S, Becker CE. Serum formate concentrations in methanol intoxication as a criterion for hemodialysis. Ann Intern Med 1986;13:343-55.

14.- Essama Mbia J], Guerit JM, Haufroid V, Hantson P. Fomizole therapy for reversal of visual impairment after methanol poisoning : a case documented by visual evoked potentials investigation. Am J Ophthalmol. 2002 Dec;|34(6):914-6.

15.- Parra Rodríguez J.C., Martinez Blanco J., Borras Cervera A., Morales Acedo M.J., Bogues Herrero M. Intoxicación por alcohol metílico. Medicina General 2002;43:292-3. 